\title{
PROPOSTA DE PLANEJAMENTO DE ATENÇÃO Á SAÚDE BUCAL DO PACIENTE COM SÍNDROME DE DOWN BASEADA EM ESTUDO DESCRITIVO BIBLIOGRÁFICO
}

\author{
PROPOSED PLANNING CARE FOR ORAL HEALTH CARE FOR PATIENTS WITH DOWN \\ SYNDROME BASED ON DESCRIPTIVE BIBLIOGRAPHIC STUDY
}
CUIDADOS DE PLANIFICACIÓN PROPUESTOS PARA EL CUIDADO DE LA SALUD DEL PACIENTE CON SÍNDROME DE ABAJO BASADO EN UN ESTUDIO BIBLIOGRÁFICO DESCRIPTIVO

Por: Mildred Ferreira Medeiros e Jhonny Oliveira Barcelos

\begin{abstract}
RESUMO
O presente estudo teve como objetivos identificar as manifestaçóes orofaciais típicas de portadores de Síndrome de Down e propor um planejamento de atenção á saúde bucal dos pacientes com Síndrome de Down - anomalia cromossomial numérica que apresenta ampla ocorrência pelo mundo. Para coleta dos dados, optou-se por realizar um estudo qualitativo descritivo e bibliográfico sobre as alteraçôes orofaciais típicas da Síndrome de Down e identificar as estratégias de atenção em saúde bucal já implementadas para tratamento de alteraçôes similares em pacientes com necessidades especiais que desenvolvem manifestaçôes orofaciais semelhantes. Para o estudo, optou-se pela metodologia qualitativa descritiva baseada em levantamento bibliográfico nas bases de dados Scientific Eletronic Online (Scielo) e MEDLINE da na Biblioteca virtual de saúde (BVS), no recorte temporal entre 2006 e 2016. Foram coletados artigos científicos em inglês e português, nos quais foi possível identificar informaçôes para conhecimento essencial do dentista: ocorrência de diferentes alteraçôes morfofuncionais sistêmicas, alteraçôes na articulação temporomandibular, dentes conoides e/ou má oclusão dental e até anodontia, prejudicando fala, mastigação e deglutição, e podendo causar fortes dores de cabeça. A partir dessas informaçóes, o plano de tratamento a longo prazo para estes pacientes especiais deve incluir higiene bucal, prótese dentária, ortodontia e estética, visando melhoria dessas condiçóes Conclui-se portanto ser necessário o conhecimento sobre o assunto pelo dentista e sugere-se que o planejamento de atenção á saúde bucal desses pacientes seja elaborado e adaptado com ampla discussão entre profissionais de saúde de outras especialidades (tais como fonoaudiólogos, psicólogos e médicos pediatras).

Palavras-chave: Síndrome de Down; Planejamento em Saúde; Anormalidades dentárias; Manifestaçôes Bucais; Odontologia.
\end{abstract}

\begin{abstract}
The present study aimed to identify the typical orofacial manifestations of patients with Down Syndrome and to propose an oral health care plan for patients with Down Syndrome - numerical chromosomal anomaly that is widely observed worldwide. For data collection, we chose to perform a qualitative descriptive and bibliographic study on the typical orofacial changes of Down Syndrome and identify the oral health care strategies already implemented to treat similar changes in patients with special needs who develop orofacial manifestations. similar. For the study, we chose the descriptive qualitative methodology based on a bibliographic survey in the Scientific Electronic Online (Scielo) and MEDLINE databases of the Virtual Health Library (VHL), in the timeframe between 2006 and 2016. Scientific articles were collected from English and Portuguese, in which it was possible to identify information for the dentist's essential knowledge: occurrence of different systemic morphofunctional alterations, temporomandibular joint alterations, conoidal teeth and / or dental malocclusion and even anodontics, impairing speech, chewing and swallowing, and may cause severe headaches. From this information, the long-term treatment plan for these special patients should include oral hygiene, dental prosthesis, orthodontics and aesthetics, aiming at improving these conditions. Therefore, it is concluded that the dentist's knowledge of the subject is necessary and it is suggested that The planning of oral health care of these patients is elaborated and adapted with wide discussion among health professionals from other specialties (such as speech therapists, psychologists and pediatricians).
\end{abstract}

Keywords: Down Syndrome; Health Planning; Tooth abnormalities; Oral Manifestations; Dentistry.

\section{RESUMEN}

El presente estudio tuvo como objetivo identificar las manifestaciones orofaciales típicas de los pacientes con Síndrome 


\section{2 | O TRÁFICO DE SERES HUMANOS PARA EXPLORAÇÃO SEXUAL E A QUESTÃO DO CONSENTIMENTO DA VÍTIMA E SUA VULNERABILIDADE}

de Down y proponer un plan de salud bucal para pacientes con Síndrome de Down: anomalía cromosómica numérica que se observa ampliamente en todo el mundo. Para la recopilación de datos, elegimos realizar un estudio cualitativo descriptivo y bibliográfico sobre los cambios orofaciales típicos del Síndrome de Down e identificar las estrategias de atención de salud oral ya implementadas para tratar cambios similares en pacientes con necesidades especiales que desarrollan manifestaciones orofaciales. similar Para el estudio, elegimos la metodología cualitativa descriptiva basada en una encuesta bibliográfica en las bases de datos científica electrónica electrónica en línea (Scielo) y MEDLINE de la Biblioteca Virtual en Salud (BVS), en el período comprendido entre 2006 y 2016. Se recopilaron artículos científicos de Inglés y portugués, en los que fue posible identificar información para el conocimiento esencial del dentista: aparición de diferentes alteraciones morfofuncionales sistémicas, alteraciones de la articulación temporomandibular, dientes conoidales y / o maloclusión dental e incluso anodoncia, que afecta el habla, mastica y traga, y puede causar dolores de cabeza severos A partir de esta información, el plan de tratamiento a largo plazo para estos pacientes especiales debe incluir higiene bucal, prótesis dental, ortodoncia y estética, con el objetivo de mejorar estas condiciones. Por lo tanto, se concluye que el conocimiento del dentista sobre el tema es necesario y se sugiere que La planificación de la atención de la salud oral de estos pacientes se elabora y adapta con una amplia discusión entre profesionales de la salud de otras especialidades (como logopedas, psicólogos y pediatras).

Palabras clave: Síndrome de Down; Planificación en Salud; Anomalías Dentarias; Manifestaciones Bucales; Odontología.

\section{INTRODUÇÃO}

A Síndrome de Down é a anomalia genética que mais acomete indivíduos no mundo inteiro. O médico inglês John Longden Hyden Down, em 1866, descreveu pela primeira vez a Síndrome de Down, sendo na altura também designada de idiotia mongoliana, devido às semelhanças físicas com a raça mongólica. Mas foi apenas em 1959 que LeJeune e Jacobs demonstraram que esta Síndrome resultava da presença de um cromossomo extra no vigésimo primeiro par, daí o termo Trissomia 21, provocando uma anomalia física e mental manifestada em vários graus (Carvalho et al., 2010; González-Agüero et al., 2009; Desai, 1997; Wuo, 2007; Silva e Dessen, 2002; Oliveira et al., 2010). Segundo o Censo de 2000 do IBGE há no Brasil cerca de 300 mil indivíduos com Síndrome de Down e, anualmente nascem cerca de 8 mil brasileiros com a síndrome.

Para o diagnóstico definitivo da Síndrome de Down, faz-se necessário um estudo cromossômico, através de investigação citogenética para a identificação do cariótipo da doença (Carvalho et al., 2010). Atualmente, dispomos de alguns exames auxiliares para a detecção das condiçôes fetais, assim como a Síndrome de Down. Dentre eles temos a amniocentese seguida de cariotipagem, o exame de sangue e a ecografia (cit. in DESSEN e SILVA, 2002).

Os portadores desta Síndrome apresentam um desenvolvimento facial alterado causado em parte à hipotonia muscular, que acomete inclusive, o sistema estomatognático, hiperflexibilidade de todo o corpo (no lactente e na primeira infância) e envelhecimento precoce. Estas crianças apresentam normalmente um volume cerebral menor do que as outras crianças incluindo reduçôes no córtex parietal e no lobo temporal (CARVALHO et al., 2010; SILVA et al., 2005; MACHO et al., 2008;). Podemos observar inúmeras alteraçôes orais ao nível do palato, da língua, dos lábios, dos dentes e na saliva. As doenças de manifestaçóes orais mais prevalentes nesta Síndrome são a cárie e a doença periodontal. Os hábitos deletérios e a má oclusão devem também ser considerados como aspectos importantes na avaliação dentária desses pacientes (SANTANGELO et al., 2008; MACHO et al., 2008; CARVALHO et al., 2010). 
O presente estudo teve como principal objetivo identificar as manifestaçôes orofaciais típicas de portadores de Síndrome de Down e propor um planejamento de atenção á saúde bucal dos pacientes com Sindrome de Down - anomalia cromossomial numérica que apresenta ampla ocorrência pelo mundo. A relevância deste estudo é oferecer ao dentista uma abordagem sistemática das possibilidades clínicas que ele poderá encontrar em seus pacientes, sugerindo parâmetros para elaboração de plano de tratamento de saúde bucal para esses pacientes com necessidades especiais, capacitando-o a atender de forma plena e poderá construir um plano de tratamento que seja adequado às necessidades de seus pacientes especiais.

\section{DESENVOLVIMENTO}

Para o desenvolvimento desse estudo, optou-se por realizar uma pesquisa bibliográfica (GIL, 2008), com abordagem qualitativa e descritiva (MINAYO, 2001) baseada na técnica de análise de conteúdo. Desenvolver uma pesquisa bibliográfica, através de revisão de literatura, constitui-se em parte integral da evolução da ciência e fornece aos profissionais de qualquer área, informação, bem como, proporciona a atualização, sobre o progresso corrente da ciência e sua literatura (FIGUEIREDO, 1990).

Ainda assim, segundo Bardin (2011), a análise de conteúdo já era utilizada de Síndrome de Down e as primeiras tentativas da humanidade de interpretar os livros sagrados, tendo sido sistematizada como método apenas na década de 20. Para Bardin (2011), o termo análise de conteúdo designa:

\footnotetext{
"um conjunto de técnicas de análise das comunicaçôes visando a obter, por procedimentos sistemáticos e objetivos de descrição do conteúdo das mensagens, indicadores (quantitativos ou não) que permitam a inferência de conhecimentos relativos às condiçôes de produçấo/ recepção (variáveis inferidas) destas mensagens" (BARDIN, 2011, p. 47).
}

Godoy (1995) afirma que a análise de conteúdo, segundo a perspectiva de Bardin, consiste em uma técnica metodológica que se pode aplicar em discursos diversos e a todas as formas de comunicação, seja qual for à natureza do seu suporte. Nessa análise, o pesquisador busca compreender as características, estruturas ou modelos que estão por trás dos fragmentos de mensagens tornados em consideração.

O esforço do analista é, então, duplo: entender o sentido da comunicação, como se fosse o receptor normal, e, principalmente, desviar o olhar, buscando outra significação, outra mensagem, passível de se enxergar por meio ou ao lado da primeira.

Para o presente estudo, as informações foram obtidas mediante análise do conteúdo dos artigos científicos coletados por levantamento bibliográfico realizado em ambiente virtual baseado nos seguintes critérios de inclusão: artigos científicos completos publicados em inglês ou português e disponíveis na íntegra para acesso gratuito nas bases de dados virtuais SciElO e MEDLINE integradas á Biblioteca Virtual de Saúde (BVS), utilizando as palavras-chave Síndrome de Down, planejamento em Saúde, anormalidades dentárias, manifestaçóes bucais e odontologia. Tais palavras-chave foram utilizadas individualmente, aos pares e em combinação múltiplos, sempre restritos ao mesmo recorte 


\section{4 | O TRÁFICO DE SERES HUMANOS PARA EXPLORAÇÃO SEXUAL E A QUESTÃO DO CONSENTIMENTO DA VÍTIMA E SUA VULNERABILIDADE}

temporal. Os critérios definidos para exclusão de textos são aqueles que não contemplam a temática do estudo os parâmetros de inclusão pré-determinados para este estudo.

A partir da coleta de informaçôes, utilizando o instrumento acima referido, foi realizada a caracterização, análise e síntese integrativa dos estudos. Foram incluídos 11 artigos para análise, de acordo com os critérios de inclusão e exclusão desta revisão.

Os pacientes com Síndrome de Down apresentam uma relativa hipotonia muscular, principalmente do sistema estomatognático. Isso pode trazer dificuldades de mastigação, deglutição, na tonicidade da língua, retenção de saliva, bem como no desenvolvimento do processo de fala e também em sua dentição (ABANTO et al., 2011).

Hábitos para-funcionais (como o bruxismo) e má oclusão bilateral também estão associados aos pacientes portadores da Síndrome de Down. São considerados aspectos importantes na avaliação médico-dental destes pacientes. As doenças da cavidade oral mais prevalentes são a cárie dental e a doença periodontal (SANTANGELO et al., 2008; MACHO et al., 2008; CARVALHO et al., 2010).

Esses pacientes, por apresentarem algum tipo de atresia dos maxilares e um subdesenvolvimento do terço médio da face, devem ser avaliados por ortodontistas e cirurgiōes ortognáticos para algum possível tipo de intervenção. Os fatores que desempenham um papel importante na má oclusão são a respiração bucal devido à associação com alteraçóes de desenvolvimento das estruturas nasais e nasofaringeas; as alteraçóes na mastigação; o bruxismo; as agenesias; o desvio da linha média maxilar; a disfunção da ATM; a erupção e esfoliação tardia de ambas as dentiçôes; a pseudomacroglossia e a hipotonia muscular. A macroglossia provoca deslocamento dos dentes e, conseqüentemente, maloclusão e hábitos bucais deletérios (COHEN 1965). Ainda como consequência da macroglossia e da hipotonicidade da língua, os lábios se apresentam freqüentemente banhados por saliva, o que leva à irritação e a fissuras nos cantos labiais, queilite angular, facilitando a instalação de processos infecciosos. (MUGAYAR, 2000). Os tipos de mal oclusão dental mais frequentes nesses pacientes são a mordida cruzada posterior, o pseudoprognatismo e mordida aberta anterior (SANTANGELO et al., 2008; MACHO et al., 2008; BARATA e BRANCO, 2010).

As crianças portadoras de Síndrome de Down podem apresentar uma cronologia de erupção dentária (decíduos e permanentes) com atrasos que variam de 6 meses a 1 ano e meio. Verifica-se, normalmente, um padrão atípico de erupção. A dentição decídua se completa por volta dos 4 ou 5 anos de idade. A retenção prolongada da dentição temporária pode levar ao apinhamento dos elementos permanentes, o que pode ser prevenido através de uma vigilância mais efetiva (BARATA e BRANCO, 2010).

Anomalias dentárias são comuns em ambas as dentições, tanto em pacientes sindrômicos ou não. Em pacientes com Síndrome de Down essa incidência é 5 vezes maior em relação a pacientes não sindrômicos. Nos pacientes com Síndrome de Down é frequente encontrar na dentição permanente agenesias dentárias, dentes supranumerários e fusóes (MACHO et al., 2013). A agenesia dos incisivos laterais é a alteração de número mais frequente na dentição temporária, enquanto na dentição permanente são os terceiros molares, os segundos pré-molares e os incisivos laterais (DESAI, 1997; MORAES et al., 2007). 


\subsection{PROPOSTA DE DIRETRIZES ESTRATÉGICAS PARA ELABORAÇÃO DE PLANO DE TRATAMENTO ODONTOLÓGICO PROPOSTO PARA PACIENTES COM SÍNDROME DE DOWN}

A literatura científica nacional e internacional disponibiliza diversos estudos que relatam as diferentes e possíveis manifestaçôes orais em pacientes portadores da Síndrome de Down. Durante a elaboração do presente estudo, buscou-se a elaboração de uma proposta de atualização sobre os principais distúrbios relacionados aos pacientes portadores de Síndrome de Down e como deveria ser elaborado o plano de tratamento para o atendimento desses pacientes pelo dentista, conforme quadro a seguir:

Tabela: Sugestão de estratégias para planejamento de tratamento odontológico de pacientes com Síndrome de Down

- Observar alteraçôes possíveis na odontogênese do paciente com exames da cavidade bucal e radiológico de imagem craniofacial (ex: raio x panorâmico e periapical).

- Orientar familiares e demais responsáveis pelo paciente sobre a necessidade de manutenção da higiene bucal de rotina e visita periódica ao dentista, possibilitando acompanhamento e diagnóstico precoce de quaisquer alteraçóes na saúde bucal (doença cárie e periodontal), ensinando técnicas adequadas para escovação e uso de fio de dental;

- Incentivar o paciente e seu responsável em aperfeiçoar as atividades de desenvolvimento motor inserindo também a técnica de escovação de dentes e uso de fio dental;

- Realizar tratamento de lesôes cariosas e de quaisquer infecções periodontais, evitando complicações secundárias (ex: endocardite);

- Planejar restauraçóes dentárias e próteses elaborando um plano de reabilitação oral para o paciente (se necessário, contatar profissional de saúde que acompanha o paciente rotineiramente) e planejar uma reabilitação de saúde multiprofissional envolvendo os âmbitos odontológico (ortodontia, cirurgia, prótese e oclusão), fonoaudiólogo e otorrinolaringológico para prestar atendimento.

\section{CONSIDERAÇÓES FINAIS}

A Síndrome de Down é um transtorno cromossômico associado com um cromossomo 21 adicional ou com trissomia parcial do cromossomo 21. As manifestaçóes clínicas estão hipotonia, baixa estatura, braquicefalia, fissuras oblíquas na pálpebra, epicanto, manchas de Brushfield na íris, língua protrusa, orelhas pequenas, mãos pequenas e largas, clinodactilia do quinto dedo, ruga dos símios e deficiência intelectual moderada a grave. malformações gastrointestinais e cardíacas, aumento marcante na incidência de leucemia e o início precoce de doença de Alzheimer. 


\section{6 | O TRÁFICO DE SERES HUMANOS PARA EXPLORAÇÃO SEXUAL E A QUESTÃO DO CONSENTIMENTO DA VÍTIMA E SUA VULNERABILIDADE}

Os portadores da Síndrome de Down apresentam inúmeras alteraçóes craniofaciais que têm potencial para causar desordens em sua saúde bucal, tais como: controle ineficaz de biofilme, apinhamentos dentários, distúrbios na articulação temporomandibular, mordida cruzada e aberta, agenesia de elementos dentários, dentes supranumerários, entre outros. Por esses motivos o acompanhamento odontológico se faz extremamente importante para a manutenção da saúde desses pacientes. A abordagem deve envolver aspectos sociais e psicológicos. O dentista deve estar motivado a estabelecer um vínculo afetivo e de confiança com seu paciente para conseguir a colaboração e o sucesso do tratamento. Além disso, os familiares e demais cuidadores desses pacientes devem estar conscientizados aos cuidados relativos à higiene oral.

O tratamento desses pacientes deve ser baseado em conceitos de saúde geral. As principais manifestaçôes orais da síndrome devem ser de conhecimento do dentista, para que haja a elaboração de um plano de tratamento que se adeque ao quadro do paciente, tendo em vista o aumento da qualidade de vida desses indivíduos.

Faz-se necessária também uma abordagem de saúde multidisciplinar, elaborada com a colaboração de profissionais de saúde de diferentes especialidades, para que haja um bem estar em todas as áreas do desenvolvimento, visando o aumento da qualidade de vida desses pacientes.

\section{REFERÊNCIAS}

ALMEIDA, SOLANGE MARIA Beltrame, Mônica; Boscolo, Frab Norberto; Manzi, Flávio Ricardo. Estudo da articulação temporomandibular em portadores de Síndrome de Down. Rev. odonto ciênc;23(1):15-19, jan.-mar. 2008.

ANDERSSON, MAUD ELS-MARIE \& BILLAUD FERAGEN, KRISTIN \& MIKALSEN, DANIEL \& KAUL, JULI \& HOLLA, THOMAS \& FILIP, CHARLES. Bilateral Hypodontia in Children With Pierre Robin Sequence.. The Cleft palate-craniofacial journal: official publication of the American Cleft Palate-Craniofacial Association. 52. 10.1597/AAID-JOI-D-11-00190, 2014.

BARATA, LÍVIA FERNANDES \& BRANCO, ANETE. Distúrbios de fala na Síndrome de Down .Rev. CEFAC. Jan-Fev; 12(1):134-139, 2010.

BARDIN, Laurence. Análise de conteúdo. São Paulo: Edições 70. 2011.

COHEN, M. MICHAEL \& WINER, RICHARD A. Dental and facial characteristics in Down'ssyndrome (Mongolism). J. Dent. Res., Alexandria, v.44, n.1, p.197-208, 1965.

E.-M. M. ANDERSSON ET AL. Bilateral hypodontia is more common than unilateral hypodontia in children with Down syndrome: a prospective population-based study.

DESAI RS \& SHAH NP. Multiple supernumerary teeth in two brothers: a case report. J Oral Pathol Med. Sep;27(8):4113, 1998.

FIGUEIREDO, Nice. Da importância dos artigos de revisão de literatura. R. bras. Bibliotecon. e Doc., São Paulo, 23(1/4):131-135, jan./dez. 1990.

GIL, Antonio Carlos. Métodos e técnicas de pesquisa social. 6. ed. São Paulo: Atlas, 2008.

GODOY, Arilda Schmidt. Pesquisa qualitativa: tipos fundamentais. Revista de Administração de Empresas, 35(4), 65-71, 1995. 
HIBA A IBRAHIM, AMAL H ABUAFFAN. Prevalência de maloclusão e necessidade de Tratamento Ortodôntico em indivíduos sudaneses portadores de síndrome de Down. 2015.

IBRAHIM ,Hiba A., ABUAFFAN, Amal H. Prevalência de maloclusão e necessidade de Tratamento Ortodôntico em indivíduos sudaneses portadores de síndrome de Down. 2015.

MACHO V, PALHA M, MACEDO AP, RIBEIRO O, ANDRADE C. Comparative study between dental caries prevalence of Down syndrome children and their siblings. Spec Care Dentist,Jan-Feb;33(1):2-7. 2013.

MESQUITA, Teresa M. da S. M. O Paciente Odontopediátrico com Síndrome de Down em Clínica Dentária. Porto, 2014.

MINAYO, Maria Cecília de Souza (Org.). Pesquisa Social. Teoria, método e criatividade. 18 ed. Petrópolis: Vozes, 2001.

MORAES, Mari Eli Leonelli de et al . Dental anomalies in patients with down syndrome. Braz. Dent. J., Ribeirão Preto, v. 18, n. 4, p. 346-350, 2007.

MUGAYAR LLR. Pacientes portadores de necessidades especiais: manual de odontologia e saúde oral. São Paulo: Pancast, p. 13-46. 2000.MUGAYAR

RAMIREZ-YAÑEZ, GERMAN \& FARIA, PAULO. Early Treatment of a Class II, Division 2 Malocclusion with the Trainer for Kids (T4K): A Case Report. The Journal of clinical pediatric dentistry. 32. 325-9, 2008.

SANTANGELO CN, GOMES DP, VILELA LO, DEUS TS, VILELA VO, SANTOS EM. Avaliação das características bucais de pacientes portadores de síndrome de Down da APAE de Mogi das Cruzes - SP. ConScientiae Saúde 2008 7(1):29- 34 .

SANTOS, Márcio José Possari ; SANGALLI, J. ; FERREIRA, A. C. R. M. ; SILVA, V. C. ; Aguiar,SMHCA . Incidência e características bucais de gêmeos monozigóticos, portadores da Síndrome de Down, considerando os fatores ambiental e comportamental. Revista Odontológica de Araçatuba , v. 30, p. 57-62, 2009.

SCHUTZ, ALINE \& UTUMI, ANTONIETA \& IGNÁCIO, SÉRGIO \& BRANCHER, JOÃO \& FREGONEZE, ANDRÉA. Análise sialométrica em indivíduos portadores da síndrome de Down. Archives of Oral Research. 9. 165. 10.7213/archivesoforalresearch.09.002.AO03. 2013.

SILVA, ELISÂNGELA RIBEIRO, PEREIRA MARCELISIO, JUNIOR, GERALDO GIL FAGGIONI. Anomalias dentárias - agenesias e dentes supranumerários- uma revisão bibliográfica. Biosci. J., Uberlândia, v. 21, n. 2, p. 105113, May/Aug. 2005.

SURI, SUNJAY \& TOMPSON, BRYAN \& CORNFOOT, LYNN. Cranial base, maxillary and mandibular morphology in Down Syndrome. The Angle orthodontist. 80. 861-9. 2013.

SURI, SUNJAY \& TOMPSON, BRYAN \& ATENAFU, ESHETU. Prevalence and patterns of permanent tooth agenesis in Down Syndrome and their association with craniofacial morphology. The Angle orthodontist. 81. 260-9. 2011. 